\title{
Dynamic behavior of direct spring loaded pressure relief valves in gas service: model development, measurements and instability mechanisms ${ }^{\text {t3 }}$
}

\author{
C.J. Hös ${ }^{\mathrm{a}}$, A.R. Champneys ${ }^{\mathrm{b}}$, K. Paul ${ }^{\mathrm{c}}$, M. McNeely ${ }^{\mathrm{c}}$ \\ ${ }^{a}$ Department of Hydrodynamic Systems, Budapest University of Technology and \\ Economics, 1111 Budapest, Müegyetem rkp. 3. Budapest, Hungary \\ ${ }^{b}$ Department of Engineering Mathematics, University of Bristol, Queen's Building Bristol \\ BS8 1TR, UK \\ ${ }^{c}$ Pentair Valves and Controls, 3950 Greenbriar Drive, Stafford, TX 77477, USA
}

\begin{abstract}
A synthesis of previous literature is used to derive a model of an in-service direct-spring pressure relief valve. The model couples low-order rigid body mechanics for the valve to one-dimensional gas dynamics within the pipe. Detailed laboratory experiments are also presented for three different commercially available values, for varying mass flow rates and length of inlet pipe. In each case, violent oscillation is found to occur beyond a critical pipe length, which may be triggered either on valve opening or closing. The test results compare favorably to the simulations using the model. In particular, the model reveals that the mechanism of instability is a Hopf bifurcation (flutter instability) involving the fundamental, quarter-wave pipe mode. Furthermore, the concept of the effective area of the valve as a function of valve lift is shown to be useful in explaining sudden jumps observed in the test data. It is argued that these instabilities are not alleviated by the $3 \%$ inlet line loss criterion that has recently been proposed as an industry standard.
\end{abstract}

Keywords:

pressure-relief valve, gas dynamics, instability, quarter-wave, Hopf bifurcation, flutter, chatter

\footnotetext{
औShort title: Dynamics of gas pressure relief valves
} 


\section{Contents}

1 Introduction 3

2 Model development $\quad 7$

2.1 Valve body dynamics . . . . . . . . . . . . . . 8

2.2 Reservoir dynamics and discharge flow rate . . . . . . . . . . 9

2.3 Pipeline dynamics . . . . . . . . . . . . . . . . . . . . . 12

2.4 Solution technique . . . . . . . . . . . . . . . . . 13

3 Experimental results and model validation $\quad 13$

3.1 Experimental set-up . . . . . . . . . . . . . . 14

3.2 Test results . . . . . . . . . . . . . . . . . . . . . . . 15

3.3 Comparison with simulations . . . . . . . . . . 20

4 Identification of instability mechanisms 21

4.1 Valve jumps . . . . . . . . . . . . . . . . 21

4.2 Flutter and chatter . . . . . . . . . . . . . . . . 24

4.3 Cycling and the $3 \%$ inlet pressure loss criterion . . . . . . . 24

4.4 Stability charts . . . . . . . . . . . . . . 28

5 Summary and outlook 30 


\section{Introduction}

This paper summarizes and extends recent scientific investigations into the mechanisms of instability in pressure relief valves (PRVs) and considers their implications for practical operation. The overall aim is to develop a new comprehensive understanding of the issues that affect valve stability in operation, in order to influence a new set of design guidelines for their operation and manufacture. In particular we shall combine theoretical model studies with tests of fully instrumented valves within representative pipe geometries. This paper will focus specifically on direct spring-loaded PRVs in gas service, particularly considering the combined effect of the valve dynamics with acoustic pressure waves within its inlet pipe.

A considerable amount of scientific literature has been published on the description and analysis of valve systems. Green and Woods (1973) provided the first comprehensive discussion of the possible causes of valve instabilities, suggesting that they can be induced as a result of five different effects: the interaction between the poppet and other elements, flow transition from laminar to turbulent during opening and closing, a negative restoring force, hysteresis of the fluid force, and fluctuating supply pressure. This paper shall focus on the first of these, specifically instability due to interaction between the valve and the inlet pipe (although, as we shall see, this can also be interpreted as an effective negative restoring force on the valve provided by an acoustic wave). Instabilities due to the other four effects identified by Green and Woods have been analyzed by a number of other authors (Kasai, 1968; McCloy and McGuigan, 1964; Madea, 1970a,b; Nayfeh and Bouguerra, 1990; Vaughan et al., 1992; Moussou et al., 2010; Beune, 2009; Song et al., 2011). Conventional PRVs subject to built-up back pressure have also been widely investigated (Francis and Betts, 1998; Chabane et al., 2009; Moussou et al., 2010). In this paper we do not consider effects of downstream piping. Oscillations in other valve systems have also been studied, e.g. in plug valves (D'Netto and Weaver, 1987), compressor valves (Habing and Peters, 2006), ball valves (Nayfeh and Bouguerra, 1990), pilot-operated two-stage valves (Botros et al., 1997; Zung and Perng, 2002; Ye and Chen, 2009) and control valves (Misra et al., 2002). Again, such studies go beyond the scope of the present work.

The first serious discussion of self-excited instabilities of poppet valves emerged in the 1960s. Funk (1964) discussed the influence of valve chamber volume and pipe length within a hydraulic circuit on the stability of a pop- 
pet valve. He found that such valves are inclined to become unstable at a critical frequency that coincides with the fundamental vibratory mode of the pipeline. Moreover, the severity of the instability increases with the length of the pipe. Kasai (1968) developed this analysis by deriving equations of motion for such a poppet valve and inlet piping system. Based on linear stability analysis, he established formulas for predicting instability in the valve. The results were shown to be in broad agreement with experiments. A similar configuration was studied by Thomann (1976) who found that the valve motion can couple to the acoustic oscillation of the pipe, leading to amplified oscillation of the system. He also developed analytical criteria for the loss of stability, finding good agreement with experiments. Later, MacLeod (1985) developed a model that includes gas dynamical issues such as choked flow capable of predicting the region of stable operation of a simple spring loaded PRV mounted directly onto a gas-filled pressure vessel.

In the 1990s Hayashi (1995) and Hayashi et al. (1997) carried out detailed linear and global stability analyses of a poppet valve circuit and showed representative examples of 'soft' and 'hard' self-excited vibration. They revealed that for the same conditions several pipe vibration modes can become simultaneously unstable, with the number of unstable modes increasing with pipe length. These results agree with those obtained by Botros et al. (1997) who find that for higher values of the pipe length two modes evolve in the system while for lower values of the pipe length the vibration is primarily in the fundamental, quarter-wave mode. They also found that maximum amplitude occurs when the oscillation frequency coincides with the quarter-wave natural frequency; for lower and higher values of the pipe length the amplitude decreases.

In early work by two of us (Licsko et al., 2009), we used nonlinear dynamical systems methods to analyze a low-order system of ordinary differential equations describing a simplified version of the set up used by Kasai (1968) and Hayashi et al. (1997), ignoring the effect of the pipe. Here we showed that upon reduction of the inlet flow rate, loss of stability is due to a Hopf bifurcation (also known as a flutter instability in aeroelastics) is initiated by a so-called self-excited oscillation; a dynamic instability which is present in the system even in the absence of explicit external excitation. The system was further investigated by Hös and Champneys (2012), where we elucidated the nature of grazing bifurcations in the system that underlie the onset of impacting motion between the valve and its seat, and performed detailed twoparameter continuation. At the same time, Bazsó and Hős (2013a) report 
experimental results in a hydraulic system that showed qualitative agreement to the nonlinear dynamics predicted by Licsko et al. (2009). That paper also presented a preliminary stability map that shows the frequency of the evolving self-excited vibration along the boundary of loss of stability, again for hydraulic application. Furthermore, Bazsó et al. (2013b) provide a detailed mathematical derivation of the model studied here in section 2, which extends the reduced-order model of Licsko et al. (2009) to include the more realistic effects of a downstream inlet pipe. The present paper though is the the first to compare that model with experimental data and to consider the practical application of the findings of the model. It should be noted that our model and conclusions bear similarities with that used in the study by Izuchi (2010). Our work though has far more detailed test data and we have also identified the key parameters and mechanisms affecting instability.

In parallel to the scientific literature, there has been industry-funded studies into the safe operation of pressure relief systems. For example, the American Institute of Chemical Engineering (AIChE) founded in 1976 the Design Institute for Emergency Relief Systems (DIERS) whose twin aims are the reduce pressure producing accidents and to develop new techniques to improve the design of relief systems. Meanwhile, the American Petroleum Institute (API) have funded their own internal program into the causes of PRV instability. Many of their findings are included in the draft 6th edition of API standard RP520 Part II. In particular, the standard is careful to point out the difference between valves undergoing three different types of behaviour, all of which have previously been referred to as instability. These are

1. cycling,

2. valve flutter, and

3. valve chatter.

Here, cycling refers to a valve that opens and closes multiple times during a pressure-relief event. Typically this behaviour is of low frequency $(<1 \mathrm{~Hz})$ and can be caused either by valve oversizing or inlet pressure loss causing the pressure to drop transiently, and the valve to shut. As pressure builds up again, the valve re-opens, with this chain of events happening repeatedly. In contrast, flutter is a high-frequency self-excited periodic oscillation of the valve (typically $>10 \mathrm{~Hz}$ ) that does not result in the valve completely closing off. Finally, chatter is a more violent form of rapid oscillatory motion that involves the valve repeatedly impacting with its seat at high frequency. The 
API RP520 standard is less clear on the precise causes of flutter or chattering instability mechanisms, but resonant coupling between the valve and its pipework, or instabilities being triggered from periodically shed vortices have been postulated as possible causes of flutter.

One of the aims of this paper is to explain these three phenomena in terms used in the recent scientific literature. In particular, we shall show that the onset of flutter can be regarded as a Hopf bifurcation (which is also commonly known as flutter in the aeroelastic research community). As shown in detail in simplified models (Licsko et al., 2009; Hős and Champneys, 2012), chatter often arises as the amplitude of the limit cycle resulting from a Hopf bifurcation grows to the extent that the valve body touches the valve seat. This causes a so-called grazing bifurcation that causes the onset of more violent, repeatedly impacting motion. Cycling behaviour is, as we have mentioned, better understood industrially and is not the subject of this paper per se. Nevertheless we do show in Section 4.3 below that our mathematical model is capable of reproducing this behaviour.

To avoid cycling, the API standard proposes that the line pressure loss should be less than $3 \%$ of the set pressure. However, as we shall see, this is not sufficient to prevent self-excited flutter or chatter instabilities in the valves we have tested.

The remainder of this paper is outlined as follows. First, Sec. 2 presents a mathematical model that combines the rigid-body dynamics of a direct spring valve with 1D gas dynamics within the pipe. The valve model is sufficiently complex to consider realistic valve design parameters such as set pressure and a prescribed relation between the effective valve area and the valve's lift. Then, in Sec. 3 we present a detailed validation of the model against test data performed on three different commercially available valves. In each case we run a pressure run-up and run-down event for for several different mass flow rates and inlet pipe lengths. A detailed comparison between model and data is presented, and close agreement is found for both the nature of the instabilities observed and for the flow rates and pipe lengths for which instability is triggered. This leads to a detailed discussion in Sec. 4 which identifies the possibility of dynamic jumps due to steepness of the effective area versus lift curves, and the origin of cycling, flutter and chatter. These terms are also explained using the language of nonlinear dynamical systems theory, in order to provide a link between the practical engineering literature and more scientific studies. Finally, Sec. 5 provides a summary, including some tentative conclusions on how instability may be prevented in future, 


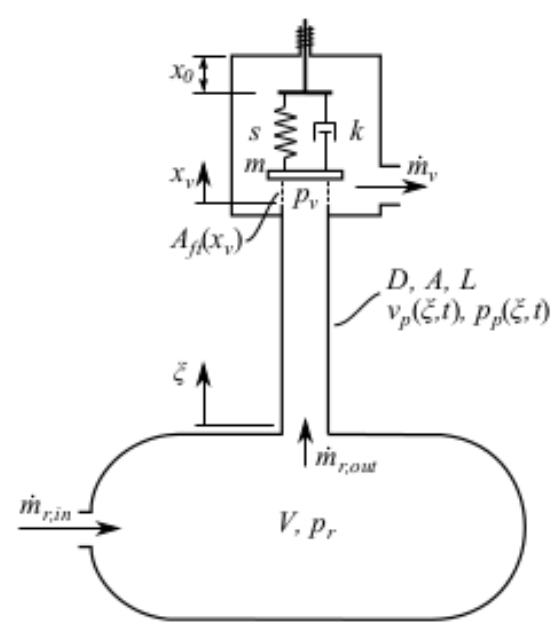

Figure 1: Definition sketch of the mathematical model

and gives an outlook to the results of future studies.

\section{Model development}

Consider the system depicted in Fig. 1 consisting of a reservoir, a pipe and a direct spring-loaded valve. The reservoir is taken to be perfectly rigid with volume $V$, pressure $p_{r}$ (that might vary in time) and temperature $T_{r}$. The mass flow rate $\dot{m}_{r \text {,in }}$ of the compressible fluid entering the reservoir is presumed either to be constant or to vary slowly when compared to other timescales present in the system (notably valve and pipe eigenfrequencies ). The change of state in the reservoir is assumed to be isentropic, that is, there is no heat exchange with the surroundings and there are no internal losses. The mass outflow from the reservoir is in general assumed to be time varying.

The flow in the long, thin pipe with diameter $D$, length $L$ and friction factor $f$ is assumed to be captured by one-dimensional unsteady gas-dynamics theory, including the effects of wall friction. Such an approach captures the inertia of the fluid, its compressibility and pressure losses, which allows for the presence of both wave effects and damping.

The valve body is modeled as a single degree-of-freedom oscillator that obeys a Newtonian equation of motion. The mass of the moving parts is $m$, the spring constant is $s$, the viscous damping coefficient is $k$. The set pressure 
is adjusted by varying the spring pre-compression $x_{0}$. The pipe pressure close to the valve body will be denoted by $p_{v}$, which is in general time-dependent. In contrast we assume constant back-pressure $p_{0}$ behind the valve.

\subsection{Valve body dynamics}

The valve itself consists of an inertial mass, the valve body, and a precompressed spring. The motion of such PRVs are usually very weakly damped. Nevertheless, we shall include some very low, nearly zero viscous damping in the model to represent the internal damping of the spring and the drag and added-mass effect of the fluid, see e.g. (Khalak and Williamson, 1997; Askari et al., 2013). The motion of the valve disk can be described as a single degree-of-freedom rigid body, with mass $m$, spring constant $s$ and viscous damping with coefficient $k$. The pre-compression of the spring will be denoted by $x_{0}$ while $x_{v}$ stands for the displacement of the valve disk. The governing equation is thus given by

$$
m \ddot{x}_{v}+k \dot{x}_{v}+s\left(x_{0}+x_{v}\right)=F_{\text {fluid }}\left(x_{v}, p_{v}\right), \quad \text { for } x_{v}>0,
$$

where a dot represents differentiation with respect to time.

The fluid force consists of two parts: pressure force and momentum force due to the deflection of the fluid jet, see the left-hand side of Figure 2. We have

$$
F_{\text {fluid }}\left(x_{v}, p_{v}\right)=p_{v} A+\dot{m}\left(v_{f, v}+v_{f, j} \cos \beta\right),
$$

where $p_{v}$ is the pressure beneath the valve, $A=D^{2} \pi / 4$ is the pipe crosssection, $\dot{m}$ is the mass flow rate through the valve, $v_{f, v}$ and $v_{f, j}$ are the mean fluid velocities in the pipe and in the jet, respectively, and $\beta$ is the jet angle. We assume that the density change is negligible between the valve end of the pipe and jet, hence $\dot{m}=\rho_{v} A v_{f, v}=\rho_{v} A_{f t}\left(x_{v}\right) v_{f, j}$ with $A_{f t}\left(x_{v}\right)=D \pi x_{v}$ being the valve flow-through area (see Figure 2 for details). Upon using the standard choked discharge equation (6) (see the next sub-section for details), we have

$$
F_{\text {fluid }}\left(x_{v}, p_{v}\right)=p_{v} A\left(1+c^{2} C_{d}^{2} \frac{A_{f t}\left(x_{v}\right)}{A}\left(\frac{A_{f t}\left(x_{v}\right)}{A}+\cos \beta\right)\right):=p_{v} A_{\text {eff }}\left(x_{v}\right)
$$

The function $A_{\text {eff }}$ will be referred to as effective area, which is defined as the force on the valve divided by the fluid pressure $p_{v}$. Introducing this quan-

tity enables us to combine the pressure and momentum force into a single 
expression, thus greatly simplifying the analysis. However, analytical estimation based on (3) requires a priori knowledge of the flow deflection angle $\beta$, which is highly non-trivial and depends not only on the valve geometry but also on the valve lift. Moreover, the effective area is likely to be a detailed function of the valve's geometry, (blow down rings, huddling chamber or shroud, etc.) and the fluid mechanics within the valve's orifices. We have found it convenient to characterize a valve by the variation of this effective area with valve lift. As we shall see, the stability of a valve can be greatly affected by the shape of this effective-area versus lift curve, which can be measured or computed by means of CFD as in Bazsó and Hős (2013b)) for each individual valve. See, for example, the right-hand side of Fig. 2 for a schematic or Fig. 11 below for an actual measured effective area curve).

Once the valve body hits the seat, i.e. when $x_{v}=0$ with $\dot{x}_{v}<0$, we apply the impact law

$$
\dot{x}_{v}^{+}=-r \dot{x}_{v}^{-},
$$

where $\dot{x}_{v}^{ \pm}$are the valve velocities immediately before and after the impact and $r$ is a coefficient of restitution.

On the other hand, if the valve is closed $x_{v}=0$ and the mean flow in the pipe is zero (so that $x_{v}=0$ and $\dot{x}_{v}=0$ ), the reservoir pressure at which the valve opens, the so-called set pressure, is given by

$$
p_{\text {set }}=\frac{s x_{0}}{A_{\text {eff }}(0)}+p_{0} \text {. }
$$

\subsection{Reservoir dynamics and discharge flow rate}

When modeling the fluid dynamics in the orifice (i.e. at the valve) and the reservoir pressure dynamics, we should keep in mind that we are trying to capture the behaviour of PRVs. Such valves are only designed to open when there is a significant pressure difference between the upstream pressure and the downstream pressure, as given by (5). It is therefore reasonable to assume that this difference is large enough for choked flow to occur. This means that flow reaches the sonic velocity at the narrowest cross section, the so-called vena contracta and hence the downstream pressure does not affect the flow rate; for details, see Zucrow and Hoffman (1976).

As an illustration to quantify when such a choked flow assumption is valid, consider the case of air, whose specific heat ratio is $\kappa=1.4$. Straightforward calculations reveal that choking occurs if the pressure ratio $\frac{p_{v}}{p_{0}}$ is greater than 1.893, which is clearly the case for pressure relief devices used in practice. 
prv_zoom_and_Aeff.pdf

Figure 2: The momentum force on the valve (left) and a typical effective area curve (right); notice the rapid increase close to zero lift which is indicative of the blowdown effect of the valve. 
Note that we do not assume that back-pressure is necessarily ambient, merely that it is constant.

The mass flow rate through the valve is given by

$$
\dot{m}=C_{d} A_{\mathrm{ft}}\left(x_{v}\right) c \sqrt{\rho_{v} p_{v}},
$$

where $C_{d}$ is the empirically derived discharge coefficient,

$$
c=\sqrt{\kappa\left(\frac{2}{\kappa+1}\right)^{\frac{\kappa+1}{\kappa-1}}}
$$

and $\kappa$ is the gas's heat capacity ratio. And $A_{\mathrm{ft}}$ is the valve's flow through area which unlike the effective area, is a pure function of geometry, which we write as

$$
A_{\mathrm{ft}}=D \pi x_{v},
$$

The imbalance between the inflow and outflow rates results in a change in the reservoir pressure. We assume that the fluid obeys the ideal gas law, $p / \rho=R T$, and that the process in the reservoir is isentropic, $p / \rho^{\kappa}=$ constant. Mass balance in the reservoir of volume $V$ therefore gives

$$
\frac{\mathrm{d} m}{\mathrm{~d} t}=V \frac{\mathrm{d}}{\mathrm{d} t}\left(\rho_{r}(t)\right)=V \frac{\mathrm{d}}{\mathrm{d} t}\left(\frac{p(t)}{R T(t)}\right)=\dot{m}_{\text {in }}-\dot{m}_{\text {out }}
$$

Given an ambient reference state $p_{0}, T_{0}$, the temperature can be related to the pressure via

$$
T(t)=T_{0}\left(\frac{\left.p_{r}(t)\right)}{p_{0}}\right)^{\frac{\kappa-1}{\kappa}}
$$

which gives

$$
\dot{p}_{r}=\kappa \frac{R T_{0}}{V}\left(\frac{p_{r}}{p_{0}}\right)^{\frac{\kappa-1}{\kappa}}\left(\dot{m}_{\text {in }}-\dot{m}_{\text {out }}\right)=\frac{a^{2}}{V}\left(\dot{m}_{\text {in }}-\dot{m}_{\text {out }}\right)
$$

with $a=\sqrt{\kappa R T}$ being the sonic velocity associated with the reservoir temperature $T$. 


\subsection{Pipeline dynamics}

The flow inside the pipe is assumed to be compressible, one-dimensional and any pressure loss can be attributed to wall friction. The gas is assumed to be ideal but the change of state is not fixed hence, besides the usual continuity and momentum equations of gas dynamics, we also need to solve an energy equation (Zucker and Biblarz, 2002; Zucrow and Hoffman, 1976). We also assume a constant pipe cross section and that the flow is adiabatic, that is there is no heat flux through the walls. Under these assumptions, the gas dynamics equations can be written in the compact vector form

$$
\frac{\partial \mathcal{U}}{\partial t}+\frac{\partial \mathcal{F}}{\partial \xi}=\mathcal{Q}
$$

with

$$
\mathcal{U}=\left(\begin{array}{c}
\rho \\
\rho v \\
\rho e
\end{array}\right), \quad \mathcal{F}=\left(\begin{array}{c}
\rho v \\
\rho v^{2}+p \\
\rho e v+p v
\end{array}\right), \quad \text { and } \quad \mathcal{Q}=\left(\begin{array}{c}
0 \\
\rho f \frac{v|v|}{2 D} \\
0
\end{array}\right)
$$

Here $\rho(\xi, t), v(\xi, t) . \quad p(\xi, t)$ and $e(\xi, t)$ are the density, velocity, pressure and energy distributions respectively, which are assumed to be functions of both the axial coordinate $\xi$ and time $t$. The overall energy $e$ of the gas can be expressed as the sum of the internal energy $c_{v} T$ and the kinetic energy $v^{2} / 2$. Assuming the gas to be ideal, we can eliminate temperature via $T=$ $p /(\rho R)$. The source vector $\mathcal{Q}$ takes the wall friction into account via a friction coefficient $f$, see Bazsó et al. (2013a) for more details of this derivation.

The boundary conditions are defined as follows. At $\xi=0$, the reservoir end of the pipe, we assume isentropic inflow into the pipe. That is, the total enthalpy at the reservoir and at the pipe entrance are assumed equal:

$$
c_{p} T_{v}=c_{p} T(0, t)+\frac{1}{2} v^{2}(0, t) .
$$

Note that this boundary condition assumes inflow into the pipe, but during cycling or violent chatter behaviour, care has to be taken to implement correct modifications to this condition (using the isentropic method of characteristics) to account for either outflow or choked flow. At $\xi=L$, the valve

end of the pipe, we set the mass flow rate leaving the pipe to be equal to the mass flow rate through the valve. Thus, we have

$$
A v(L, t) \rho(L, t)=C_{d} A_{\mathrm{ft}}\left(x_{v}\right) c \sqrt{\rho(L, t) p(L, t)} .
$$




\subsection{Solution technique}

Putting the above pieces together, the model consists of the equation of motion of the valve (1), (4), the reservoir dynamics (10) and the pipeline dynamics (11) with boundary conditions (12) and (13). Note that the system of equations is fully coupled; we do not solve for the valve motion and pipe flow separately. The coupling arises through the boundary conditions (12), (13) of the partial differential equation (PDE) system (11) and the right-hand side forcing terms of the ordinary differential equations (ODEs) (1) and (10).

The model is solved using a finite difference scheme, implemented in Matlab. In each time step $\Delta t$ the ODEs (1) and (10) are solved using a standard Runge-Kutta technique, while the PDE system solved using a standard LaxWendroff finite difference scheme (described, for example, in Cebeci et al. (2005) or Warren (1983)). The spatial step length $\Delta \xi$ is chosen to be uniform and to satisfy the CFL condition, $\Delta \xi=\max (a+|v|) \Delta t$. This criterion ensures that information propagation does not jump over any cell during one time step. Once the pipeline dynamics is updated, the valve and reservoir dynamics are also integrated from $t$ to $t+\Delta t$. Finally, the boundary conditions (12) and (13) are solved in an iterative way. Hence, the dynamics of the three elements are integrated in a fully coupled way. During a typical simulation, a minimum of 20 grid points are placed along the pipe; hence a minimum of 20 time steps are taken during one full pipe oscillation period $T=L / a$.

Experimentation with more grid points showed this choice to be suitable to resolve the gas dynamical effects in the pipe with high fidelity. Notably, wave propagation and reflections at the ends could be reliably reproduced. The computational effort required was found to be such that a typical computation took 5-10 minutes on a standard desktop PC.

\section{Experimental results and model validation}

In what follows we shall describe the results of simulations of the above equations of motion, to match experimental results on three commercially available valves, with product codes 1E2, 2J3 and 3L4. The actual parameter values used in the simulations are given in Table 1. Unless otherwise stated, the effective area $A_{\text {eff }}=D_{\text {eff }}^{2} \pi / 4$ was taken to be constant. 


\begin{tabular}{|l|c||c|c|c||l|}
\hline Quantity & Symbol & $1 \mathrm{E} 2$ & $2 \mathrm{~J} 3$ & $3 \mathrm{~L} 4$ & Units \\
\hline \hline Mass flow rate & $\dot{m}_{r, \text { in }}$ & $0-3$ & $0-15$ & $0-16$ & $\mathrm{lb} / \mathrm{s}$ \\
Pipe length & $L$ & $0-72$ & $0-72$ & $0-72$ & inch \\
Pipe diameter (nom. inner) & $D$ & 1.049 & 2.067 & 3.068 & inch \\
Effective pressure diameter & $D_{\text {eff }}$ & 0.635 & 1.6043 & 2.3493 & inch \\
Reservoir volume & $V$ & 375 & 375 & 375 & $\mathrm{ft}^{3}$ \\
Total effective moving mass & $m$ & 0.976 & 3.358 & 14.43 & $\mathrm{lb}$ \\
Spring constant & $s$ & 415 & 714 & 688 & $\mathrm{lbs} /$ inch \\
Damping coefficient & $\%$ of $k_{\text {crit }}$ & $4.9 \%$ & $2 \%$ & $1 \%$ & $\mathrm{lbs} / \mathrm{inch}$ \\
Set pressure & $p_{\text {set }}$ & 452 & 253 & 100 & $\mathrm{psi}$ \\
Coefficient of discharge & $C_{d}$ & 0.93 & 0.93 & 0.93 & - \\
Coefficient of restitution & $r$ & 0.8 & 0.8 & 0.8 & - \\
Maximum lift & $x_{\text {max }}$ & 0.204 & 0.472 & 0.770 & inch \\
Ambient temperature & $T_{0}$ & 293 & 293 & 293 & $\mathrm{~K}$ \\
Ambient pressure & $p_{0}$ & 14.7 & 14.7 & 14.7 & $\mathrm{psi}$ \\
Gas constant & $R$ & 288 & 288 & 288 & $\mathrm{~J} /(\mathrm{kgK})$ \\
Specific heat ratio & $\kappa$ & 1.4 & 1.4 & 1.4 & - \\
\hline
\end{tabular}

Table 1: Parameter values used for each of the three valves.

\subsection{Experimental set-up}

The general experimental set up used is depicted in Fig. 3. The test rig consists of a reservoir connected to the test valve via a standing pipe. The reservoir is fed with nitrogen gas through a sonic nozzle allowing constant inflow, so that upstream reservoirs (not depicted in the figure) can effectively be considered to be infinitely large. Besides direct valve displacement measurements, several pressure and temperature measurements were also taken, as indicated in the figure. All sensors were sampled at $1 \mathrm{kHz}$ with a standard desktop PC.

Each valve was tested for several different pipe lengths, chosen from among pipes of length 12, 18, 24, 48, 72 inches and at mass flow rates which represent 30,50 , and $100 \%$ of that valve's nominal flow rate.

Constant inlet mass flow rate was set with the help of a sonic nozzle. The mass rate of flow at the sonic nozzle was computed by means of the ASME Research Report on Fluid Meters (see Bean (1971)): 


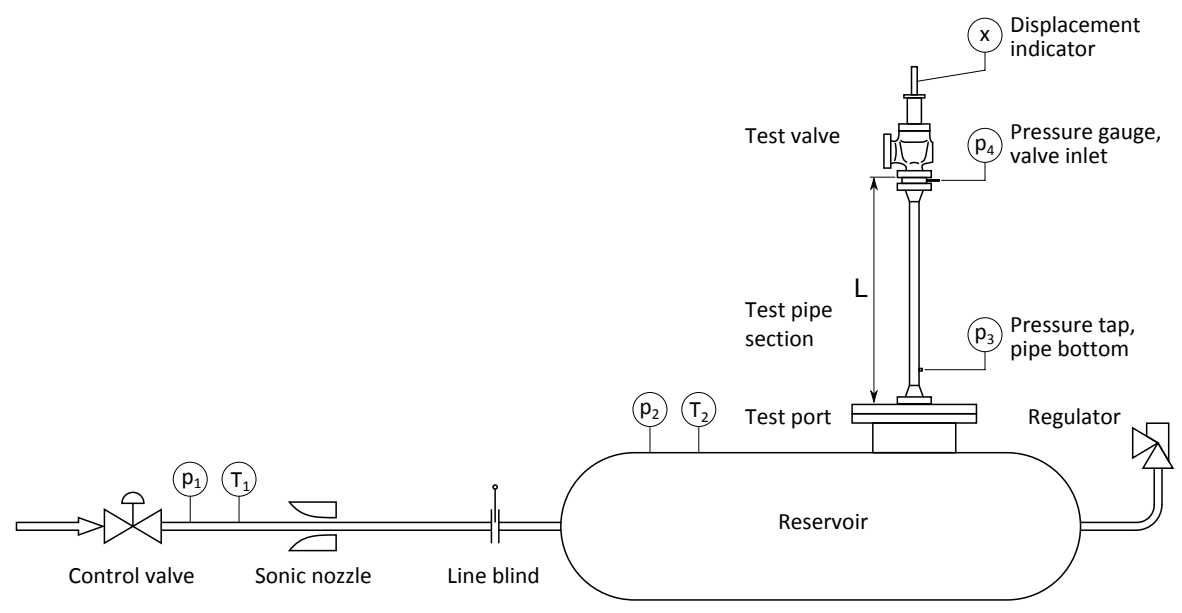

Figure 3: Schematic representation of the experimental test rig

$$
\dot{m}\left[\frac{l b_{m}}{s}\right]=\operatorname{Ca\phi }_{i}^{*}\left(\frac{\phi^{*}}{\phi_{i}^{*}}\right)\left(\frac{p_{1 \mathrm{t}}}{\sqrt{T_{1 \mathrm{t}}}}\right)
$$

where $C$ stands for the discharge coefficient (for ASME flow nozzles $C=$ 0.990), $a$ is the throat area of sonic-flow primary element $p_{1 \mathrm{t}}$ and $T_{1 \mathrm{t}}$ are the inlet stagnation pressure and temperature, respectively. $\phi_{i}^{*}$ is the sonic-flow function $\left(\phi_{i}^{*}=0.52295\right)$ and $\frac{\phi^{*}}{\phi_{i}^{*}}$ is the ratio of the real-gas sonic-flow function and the sonic-flow function, which can be obtained from tables. The actual value for nitrogen is $\frac{\phi^{*}}{\phi_{i}^{*}}=1.0130$.

During the measurements, the control valve before the sonic nozzle was opened, resulting in constant inflow to the tank and an increase to tank pressure. Once the tank pressure reached the set pressure of the valve, the PRV opened and was found to either operate in a stable manner or to become unstable to flutter. In both cases, after a few seconds the tank regulator valve was opened, which allowed the tank pressure to reduce and led to the re-closure of the valve.

\subsection{Test results}

Typical measurement results are shown in Figs. 4-7 showing examples of both stable and unstable operations. 

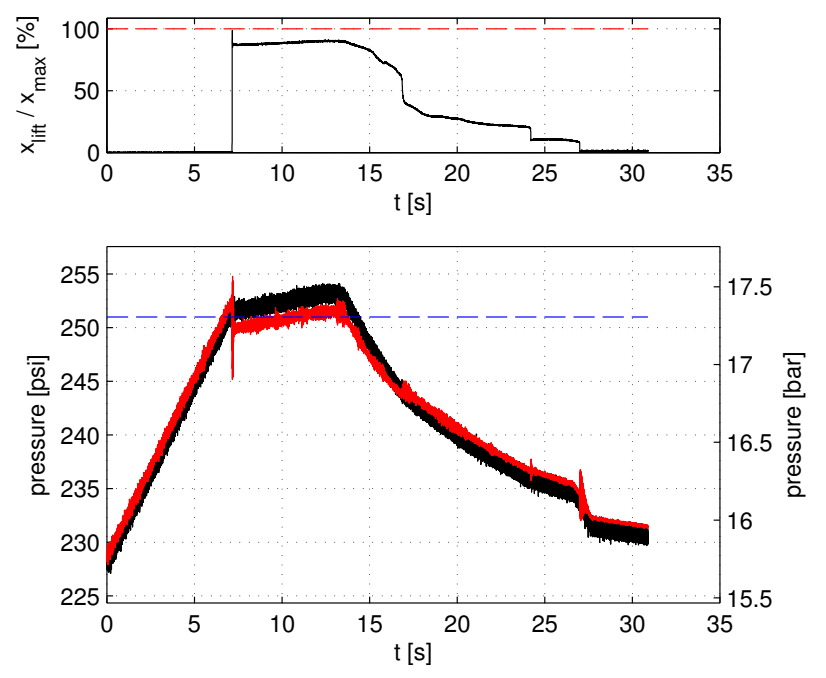

Figure 4: Example of a stable test for valve $2 \mathrm{~J} 3$ with drive pressure 500 psig and an inlet pipe of 24 inches. (Upper panel) Valve lift, depicted as percentage of maximum lift, which is represented by a dashed line. (Lower panel) pressure at the reservoir (red) and valve (black) end of the pipe.

Figures 4 and 5 show the two extremes of the observed dynamics: a stable opening-closing cycle and an unstable one, respectively. Note in the stable case Fig. 4, that even though there are no oscillations of the valve body, there are sudden 'jumps' in the valve's lift; a single 'jump up' to maximum lift on opening, and a sequence of 'jumps down' as the valve closes. This issue will be addressed in Sec. 4.1 below. Note also, that that the jumps on opening and final closing cause impulsive, rapidly decaying oscillations in the pipe that are not translated into the valve motion.

In contrast, Fig. 5 depicts a completely unstable valve cycle: after opening, the valve goes immediately unstable, and vibrates heavily. Note that these oscillations are of the most extreme kind, chatter, under the classification outlined in Sec. 1, because the valve lift reaches zero during each oscillation cycle. Each closure involves a hard impact of the valve with its seat. The resulting vibrations are strongly audible and, were it not for extra strengthening procedures that were instigated in the lab, would have likely permanently damaged the valve. Note also that the valve motion is clearly coupled to that of the pipeline and we observe severe pressure pulsations.

We have also found examples where the valve is stable on opening but 

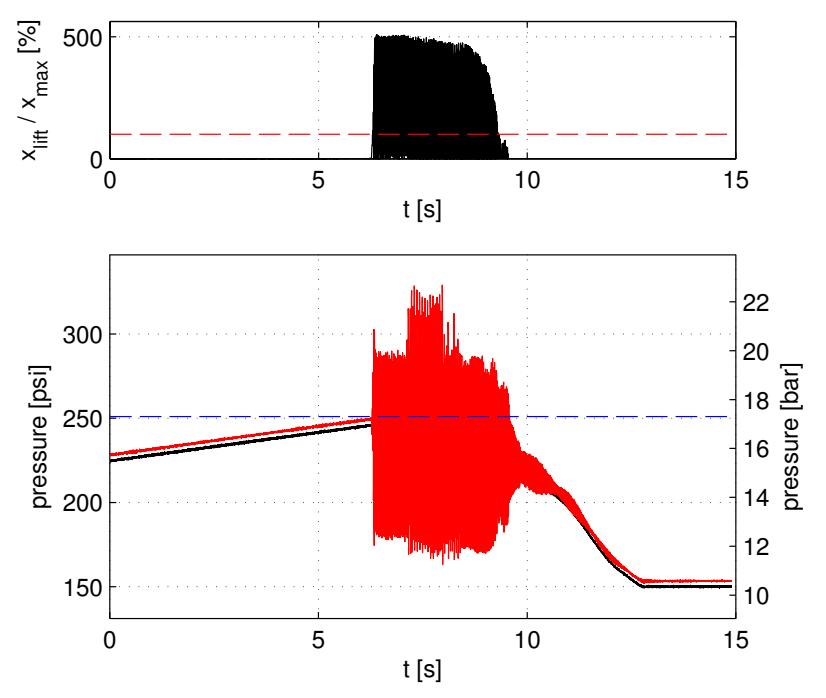

Figure 5: Similar to Fig. 4 but showing a completely unstable test for valve 2J3 with drive pressure $500 \mathrm{psig}$ and an inlet pipe of 72 inches. The unrealistically high displacement measurements are due to a lost contact between the displacement transducer and the valve shaft.

unstable on closing, see Fig. 6. Here note the initial oscillation, at around 16 seconds, can be classified as flutter, using the scheme outlined in Sec. 1 because the valve does not impact with its seat. There is a sudden transition though into chatter at around 19 seconds, at which point the oscillations became strongly audible.

Figure 7 shows another intermediate case, where a flutter instability is triggered on opening the valve at around 3 seconds, which abruptly ends at 4.5 seconds. A similar instability is then triggered on closing the valve, which apart from a brief grazing event at around 15 seconds does not develop into chatter. Note that the final large excursion into large positive lift is a measurement failure, as in Fig. 5 when the displacement transducer lost contact with the valve as the valve closed.

Figure 8 shows a close-up of valve displacement and pressure fluctuation measured over several periods of steady, limit cycle oscillation during flutter. Zooming in on these pressure fluctuations, over this short time scale, we observe that the pressure at the top of the pipe is one quarter of a cycle out of phase with that at the tank end. Moreover, the valve oscillation is half a cycle out of phase with the pressure. This data is typically of all the 

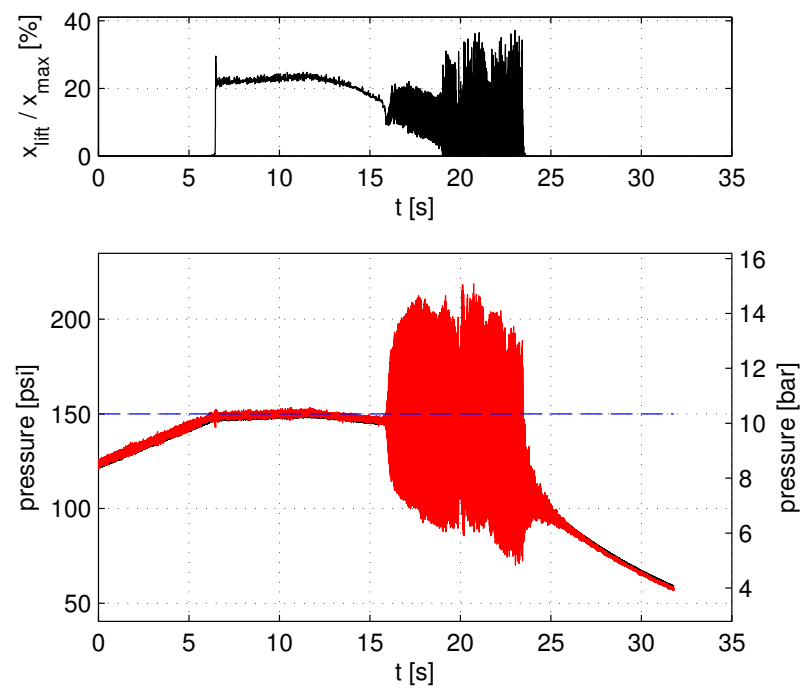

Figure 6: Similar to Fig. 4 but showing a case where the valve is stable on opening but unstable on closing. Valve type: 3L4, pipe length: $4 \mathrm{ft}, 50 \%$ of capacity flow rate.

oscillatory motion we have observed during flutter, and will prove important in Section 4 where we seek an explanation for this phenomenon. 

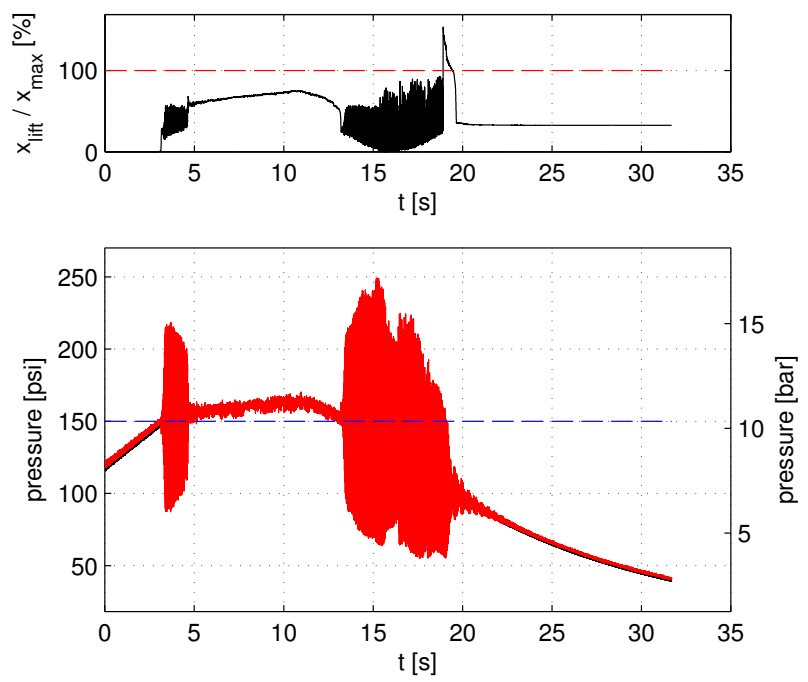

Figure 7: Similar to Fig. 4 but where the valve is stable on opening but unstable on closing. Valve type: $3 \mathrm{~L} 4$, pipe length: $6 \mathrm{ft}, 100 \%$ of capacity flow rate.
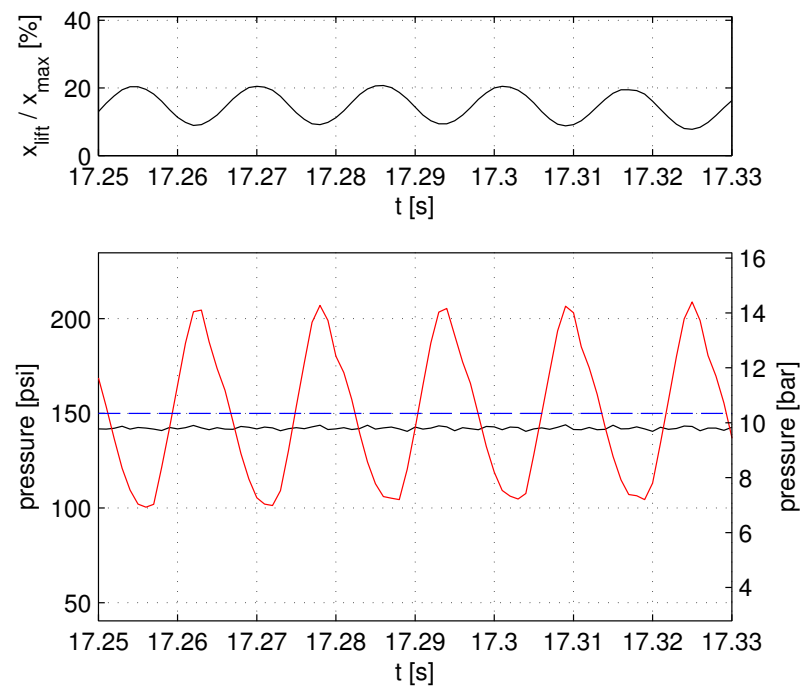

Figure 8: Close-up of valve displacement (upper panel) and pressure (bottom panel) measurements during flutter. In the pressure plot, the almost constant solid black line shows tank pressure and the solid red line shows the pressure at the valve end of the pipe; the set pressure is represented by a dashed line. (parameters: $3 \mathrm{~L} 4$ valve, $4 \mathrm{ft}$ pipe, mass flow rate: $50 \%$ of capacity) 


\subsection{Comparison with simulations}

We have performed a large number of numerical computations, which will be analyzed later in detail. At this point we only emphasize that we have been able to replicate all this behaviour using the simulation model as shown for example in Figure 9 and 10. In the simulations below, we used the effective area curve depicted in the upper left panel of Figure 11.
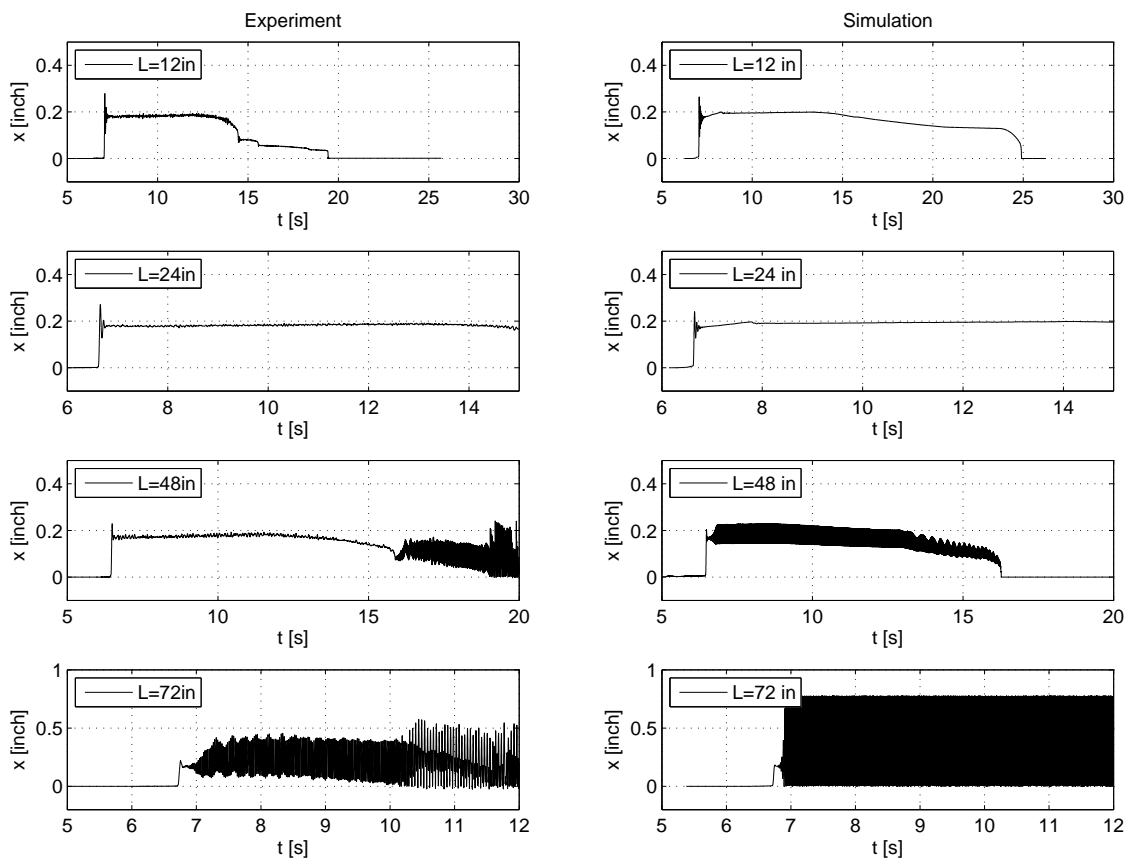

Figure 9: Comparison between experimental data and model simulation for valve size 3L4 at $50 \%$ of capacity (i.e. $6 \mathrm{lb} / \mathrm{m} / \mathrm{s}$ ).

Figure 9 shows a comparison between experiment and simulation on a range of tests for the valve 3L4 with a mass flow rate $50 \%$ of the valve's capacity rating, but for different pipe lengths. Note the strong qualitative and good quantitative comparison between the two sets of data. Note also that this has been achieved without any parameter fitting, except for taking a reasonable, ballpark estimate of valve damping $k$. Nor have we bothered with measuring and fitting an accurate effective area versus lift curve. This 
figure also highlights the general trend we have seen in both the tests and simulations; namely that for each value of mass flow rate, there is a critical pipe length beyond which instability occurs. For pipes just longer than this critical length, the amplitude of the fluttering motion grows and transitions into chatter, becoming more violent with increasing pipe length.

We have experimented with changes to how the convective terms in the equations of motion are introduced, how much pipe friction was included, with the coefficient of damping and found that each of these had a weak effect on the location of the critical pipe length at which instability occurred. The same is true of the shape of the effective area curve, although this did seem to change some of the transient dynamics close to the instability point, particular in whether instability was seen on opening, on closing or both. Changes to the coefficient of restitution obviously only affected the postchattering motion and had no influence on the location of the instability point.

Figure 10 shows similar data for a much smaller valve, the 1E2. This valve is designed to withstand much greater pressure build up. Again we see the same trends in the data and the same level of correspondence between the simulations and the test data, although we do note that the instability when it occurs appears more immediately and with larger amplitude in the simulation than the data. Careful fitting of damping parameters and effective area curves would likely produce a stronger degree of quantitative similarity.

\section{Identification of instability mechanisms}

We shall now explore our experimental and computational findings in more detail. In particular the mechanisms by which instabilities are triggered will be elucidated.

\subsection{Valve jumps}

The first issue we discuss is the possibility of a static instability (i.e. sudden jump in the valve lift without long-lasting oscillations). Such effects can be seen on valve opening in the test data in Fig. 7 where there is a jump up in valve lift on initial valve opening that triggers a flutter instability followed a second such jump that causes the instability to suddenly cease). Similar effects can also be observed on valve closing in the test and simulation data in Fig. 9 where there are two jump downs, the second of which triggers a chatter type instability for a short time before the valve closes for good. 

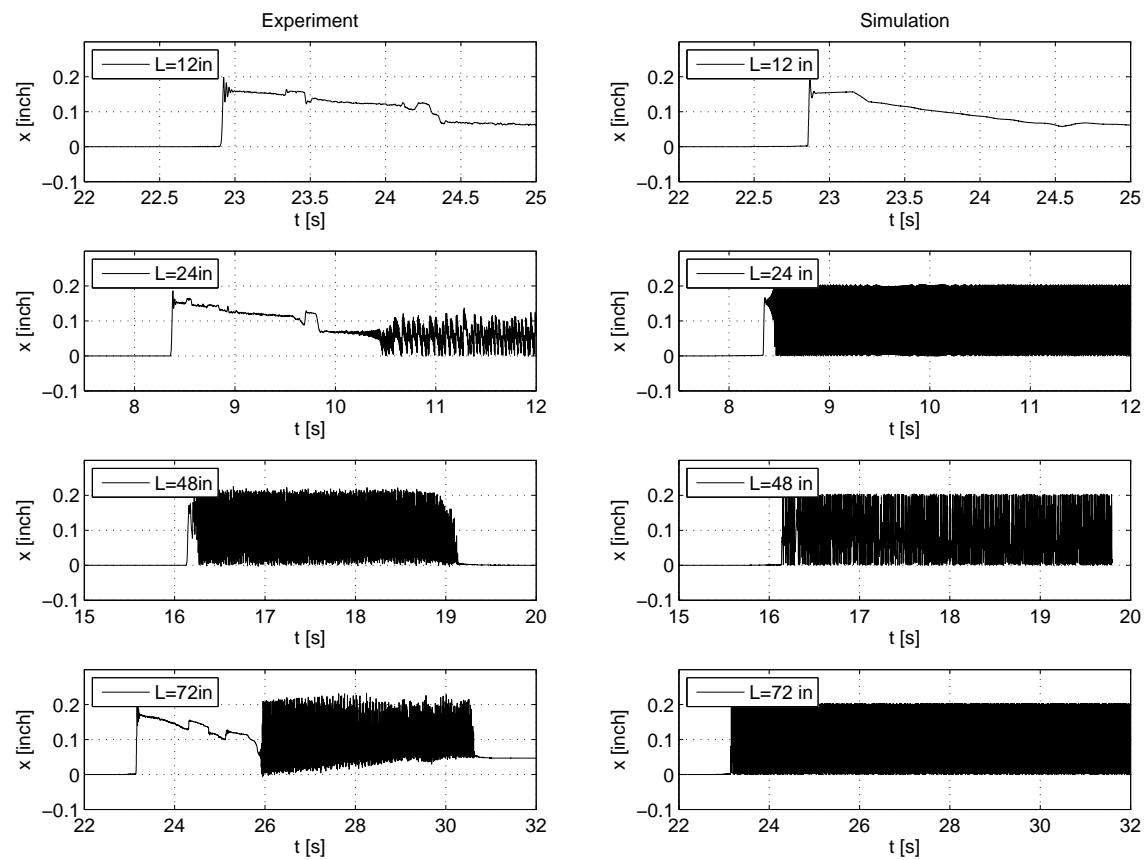

Figure 10: Comparison between experimental data and model simulation for valve size $1 \mathrm{E} 2$ at $50 \%$ of capacity (i.e. approx. $1.15 \mathrm{lb}_{\mathrm{m}} / \mathrm{s}$ ).

We have found that such effects can be explained by consideration of the shape of $A_{\text {eff }}\left(x_{v}\right)$, the effective area versus lift curve, as we now explain.

Suppose that $x_{v}^{0}$ is the static equilibrium lift for a given valve pressure $p_{v}$ and consider a small perturbation $x_{v}=x_{v}^{0}+\varepsilon$ while valve pressure $p_{v}$ remains approximately constant. This is a reasonable assumption to leading-order since pressure is constrained in practice to be no more than $10 \%$ above set pressure when the valve is fully open. Then, to leading order, $\varepsilon$ satisfies the differential equation

$$
m \ddot{\varepsilon}+\kappa \dot{\varepsilon}+s \varepsilon=A_{0}^{\prime} p_{p} \varepsilon,+\mathcal{O}\left(\varepsilon^{2}\right) \quad \text { where } A_{0}^{\prime}=\left.\frac{d A_{\text {eff }}}{d x_{v}}\right|_{x=x_{v}^{0}}
$$

where $A_{0}^{\prime}$ stands for the slope of the effective area curve evaluated at the equilibrium valve lift, see the upper panel of Figure 11, where measured data and a curve fit through that data are shown. 

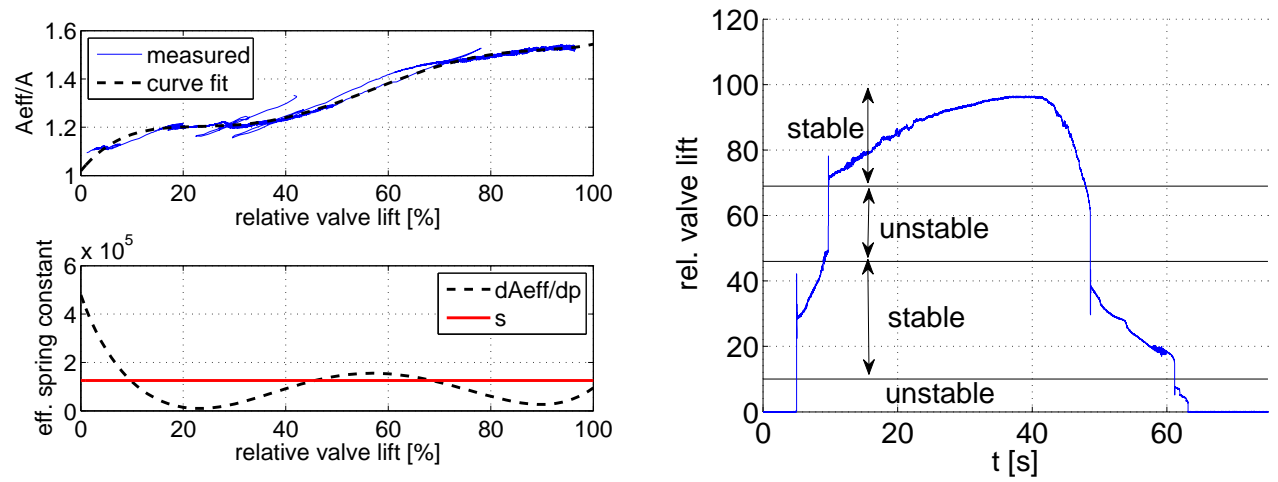

Figure 11: Measured static instability curves for the 2J3 valve and the prediction of jump instabilities. Left: upper panel - effective area curve as a function of valve lift (here $A_{1}$ stands for seat area), measured values (thin blue line) and curve fit (dashed black curve); bottom panel - effective spring constant which is calculated from the slope of the measured effective area curve (dashed black line) and spring constant (horizontal red line) as a function of the valve lift. For a given valve lift $\left(x / x_{\max }\right.$ value) the equilibrium is stable if the spring stiffness is larger than the slope of the effective area curve. Right: simulation data with for a particular run of the J23 data in which jumps can be observed for the unstable lift values predicted by the theory in the left-hand panel.

Notice from (15) that the equilibrium valve lift is stable as long as the effective spring stiffness

$$
s_{\text {eff }}=s-A_{0}^{\prime} p_{p}>0 .
$$

This is because the term $s_{\text {eff }}$ multiplies the linear displacement term $\varepsilon$ in the equation. However, if $s_{\text {eff }}$ becomes negative (i.e. the effective area curve is too steep), then we have a negative stiffness term (multiplying $\varepsilon$ ). Hence the equilibrium lift becomes unstable. At such a point we would have a so-called fold (or saddle-node) bifurcation, see e.g. Kuznetsov (2004) which would result in a jump to another (stable) equilibrium position. Note that for the results in Fig. 11, the unstable portion is between about $42 \%$ and $65 \%$ lift for this valve.

Note that this prediction is borne out in the experimental measurements, see the right-hand panel in Fig: 11, which shows dynamic jumps occurring in a run in which a valve opens and then closes. Note that the jumps up and jumps down occur approximately as the displacement enters the region where the analysis predicts instability, that is between about $42 \%$ and $65 \%$ valve lift. 


\subsection{Flutter and chatter}

The flutter instability bears all the hallmarks of a Hopf bifurcation, see e.g. Kuznetsov (2004). Such instabilities are characterized, upon quasi-static parameter variation, by a transition to negative damping. In the so-called supercritical version of the bifurcation, a stable limit cycle motion ensues, whose amplitude grows like the square root of the distance of the parameter from its bifurcation point. The period of the limit cycle is related to the imaginary part (frequency) of the eigenvalue of the linearized system at the instability point.

We have conducted a careful analysis of the valve motion and the pressure dynamics, notably extracted the frequency content of the experimentally measured valve displacement signals with the help of the fast Fourier transform (FFT). As shown in Table 2, once the valve goes unstable to flutter, the dominant frequencies obtained from both the displacement and pressure signals are close to the pipe quarter-wave frequency, i.e. $f_{q w}=4 L / a$ with $a$ being the sonic velocity, and are a long way from the valve spring's own resonant frequency. Note that the measured dominant frequencies are slightly lower than the quarter-wave frequency, which is due to the inertial effects on the end of the pipe as explained e.g. in Ih (1993).

This mode of instability is also consistent with the recordings shown in Fig. 8 where we see the motion at the two ends of the pipe are a quarter of a cycle out of phase with each other. Although not presented here, the $1 \mathrm{E} 2$ and the 3L4 valve measurements resulted in similar results. Hence we conclude that close to the critical pipe length, when the valve starts to flutter, the mode of vibration that is involved in the Hopf bifurcation is that which corresponds to a quarter standing wave in the pipe. Note that this is a coupled mode that involves both the fluid and the valve; see for example Fig. 8 where the valve oscillates at the same frequency as the fluid pressure, albeit half a cycle out of phase as one would expect from physical principles.

This conclusion that the mode of instability at the Hopf bifurcation involves a quarter standing wave is confirmed by the simulations. Figure 12 shows typical results for a pipe length just beyond that for which instability is triggered. This again confirms that the valve and the fluid are oscillating at the same frequency, and the mode shape inside the pipe is clearly apparent.

\subsection{Cycling and the 3\% inlet pressure loss criterion}

It is well known that over-sized valves are susceptible to cycling behaviour. Figures 13 and 14 show simulation results for the $3 \mathrm{~L} 4$ valve at $6 \%$ of its 

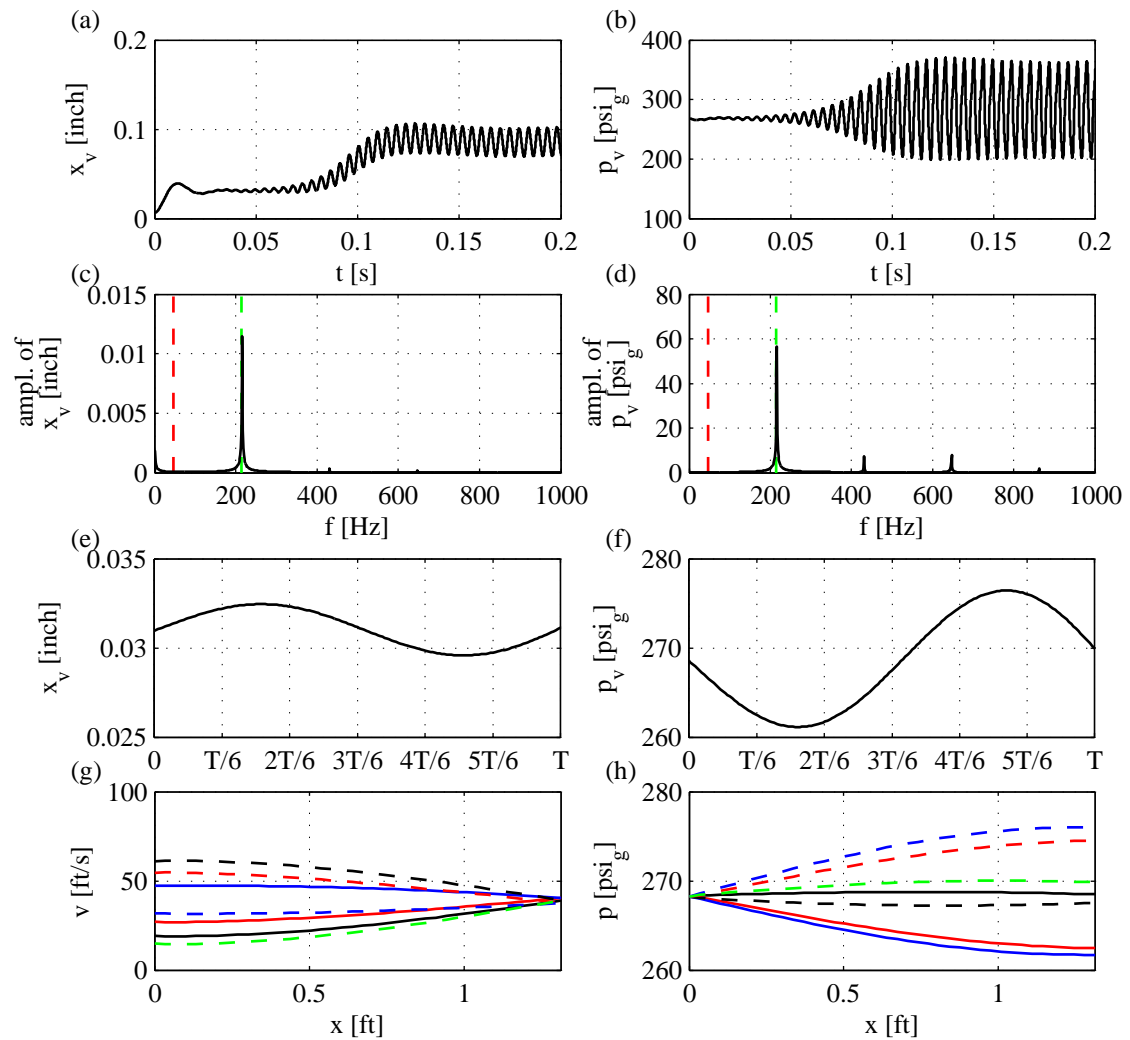

Figure 12: Simulated instability for $2 \mathrm{~J} 3$ valve with $L=24$ inch and $30 \%$ of capacity (i.e. close to the stability border). (a), (b) Time history of valve lift and pressure at valve just after opening. (b),(c) Same information plot over one oscillation cycle. (d),(e) Mode shape of the fluid flow and pressure in the pipe over half a cycle. Different line types in each plot represent the same time instant, which are plot at intervals of $1 / 8$ of one period of oscillation. 


\begin{tabular}{|c|c||c|c|}
\hline L [inch] & $\begin{array}{c}\text { mass flow rate } \\
{[\mathrm{lb} / \mathrm{s}]}\end{array}$ & $\begin{array}{c}\text { dominant freq., } \\
\text { experiment [Hz] }\end{array}$ & $\begin{array}{c}\text { quarter-wave } \\
\text { frequency [Hz] }\end{array}$ \\
\hline \hline \multirow{3}{*}{24} & 5.4 & stable & - \\
\cline { 2 - 4 } & 9.2 & stable & - \\
\cline { 2 - 4 } & 13.2 & stable & - \\
\hline \multirow{3}{*}{48} & 5.4 & 85.62 & 96.05 \\
\cline { 2 - 4 } & 9.2 & 83.68 & 96.23 \\
\cline { 2 - 4 } & 13.2 & 83.37 & 95.98 \\
\hline \multirow{3}{*}{72} & 5.4 & 62.34 & 64.13 \\
\cline { 2 - 4 } & 9.2 & 61.98 & 64 \\
\cline { 2 - 4 } & 13.2 & 66.16 & 64.14 \\
\hline
\end{tabular}

Table 2: Frequency content of the valve displacement signal (measurements) versus the calculated pipe quarter-wave frequency (from the model) in the case of the 2J3 measurements. Note that the valve eigenfrequency is $45.6 \mathrm{~Hz}$.

maximum flow rate. In the first case, for a short pipe, the valve opens into a stable regime. The pressure relief is such that too little fluid escapes during the initial valve opening that the pressure continues to build up again in the tank. This then causes repeat openings, in a periodic very low frequency cycle. Such behaviour is not intended but is not likely to be damage inducing.

In contrast, Fig. 14 shows the same effect, but for a much longer pipe. Here when the valve opens, it is into a regime that is well into the instability region, and chatter immediately ensues. Each time the valve opens there is a period of rapid chattering behaviour. This behaviour is likely to be damage inducing.

Cycling can also occur at higher flow rates, when the inlet piping pressure loss is too much. It has been recommended in the API standard RP520 that this pressure loss should be kept to under $3 \%$ of set pressure. The critical pipe length corresponding to the ' $3 \%$ rule' can easily be calculated, under standard assumptions about frictional losses of pipes of a given parameter. We briefly recall that the pressure loss due to wall friction in a straight pipe is given by (see Zucker and Biblarz (2002) for details)

$$
\Delta p^{\prime}=\lambda \frac{L}{D} \frac{\rho}{2} v^{2}=\lambda \frac{L}{D} \frac{\rho}{2} \frac{\dot{m}^{2}}{\rho^{2} A^{2}},
$$

from which it is straightforward to find the critical pipe length $L$ for which 

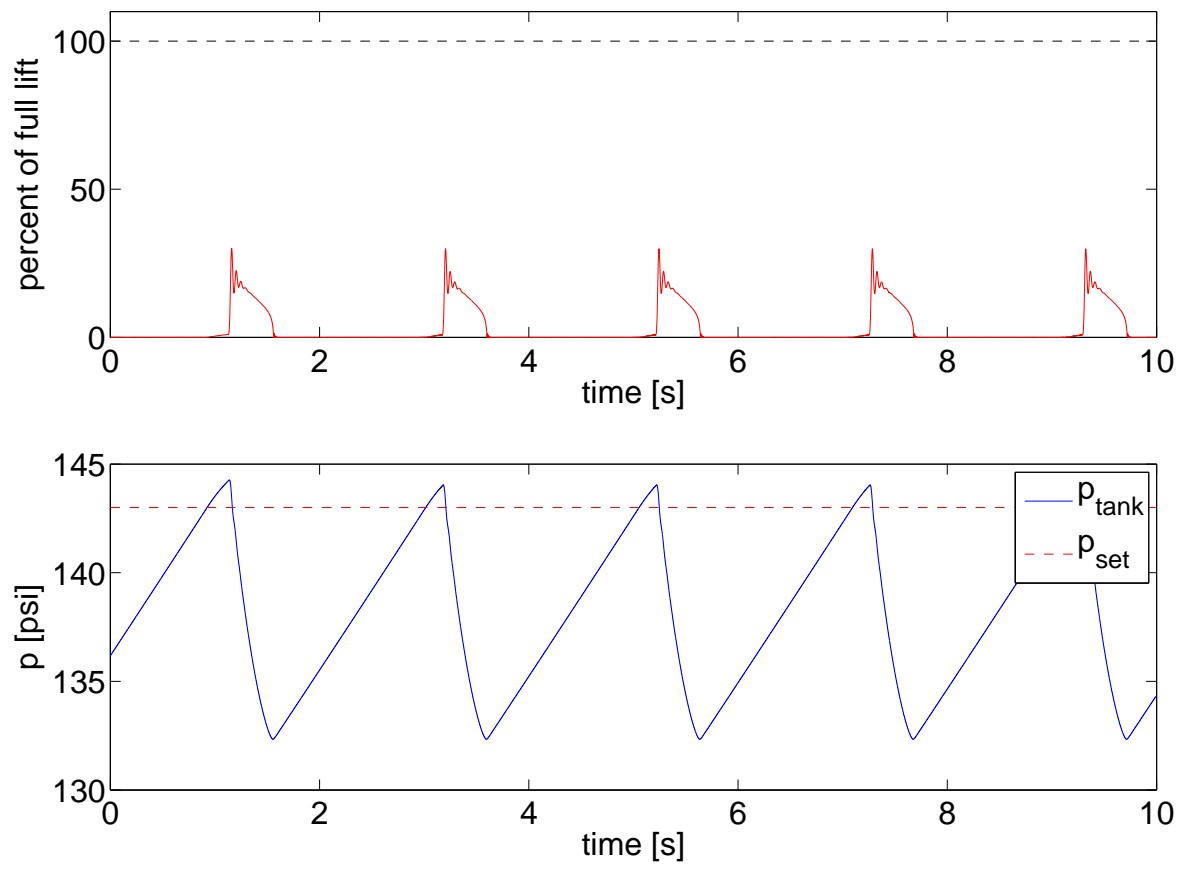

Figure 13: Simulation of cycling behaviour for the 3L4 valve with a 24 inch pipe at $6 \%$ of its maximum mass flow rate. 

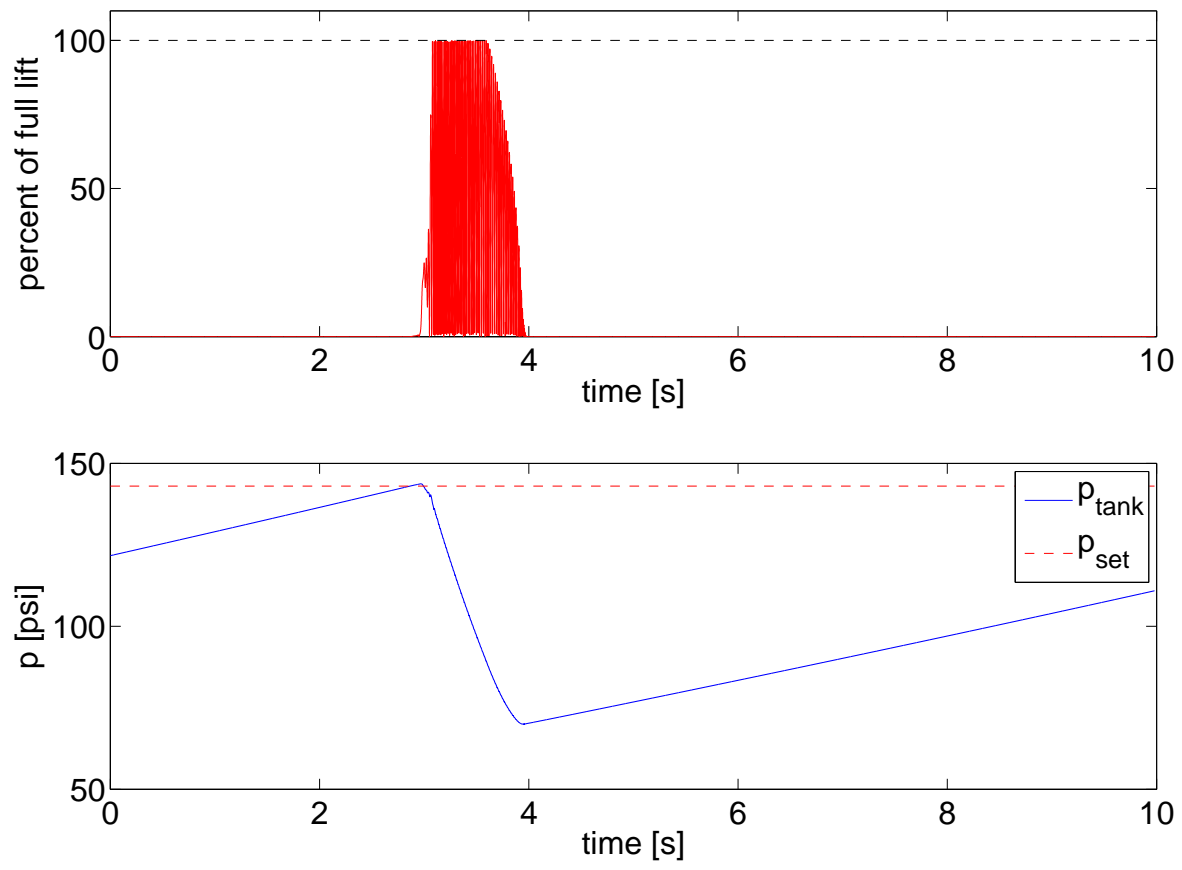

Figure 14: Similar to Figure 13 but for a 72 inch inlet pipe.

$\Delta p^{\prime}=0.03 \times p_{\text {set }}$. Specifically, we used $\lambda=0.02$ for Darcy friction factor, which is being four times larger than the Fanning friction factor (Zucker and Biblarz, 2002). This critical pipe length is plot on top of the stability maps for each of the pipes which we present in the following subsection.

\subsection{Stability charts}

Figures 15, 16 and 17 present a summary of the stability information we have found for each of the three valves. The solid magenta curve in each figure shows the flutter boundary as computed using the simulation. This was computed by visual inspection of the output of simulation runs each mass flow rate at intervals of $0.1 \mathrm{lb}_{m} / \mathrm{s}$. A simple bisection method was then used to find the critical pipe length at which the Hopf bifurcation is observed. This curve therefore represents the transition from stability (lower pipe lengths) to instability (longer pipes). 


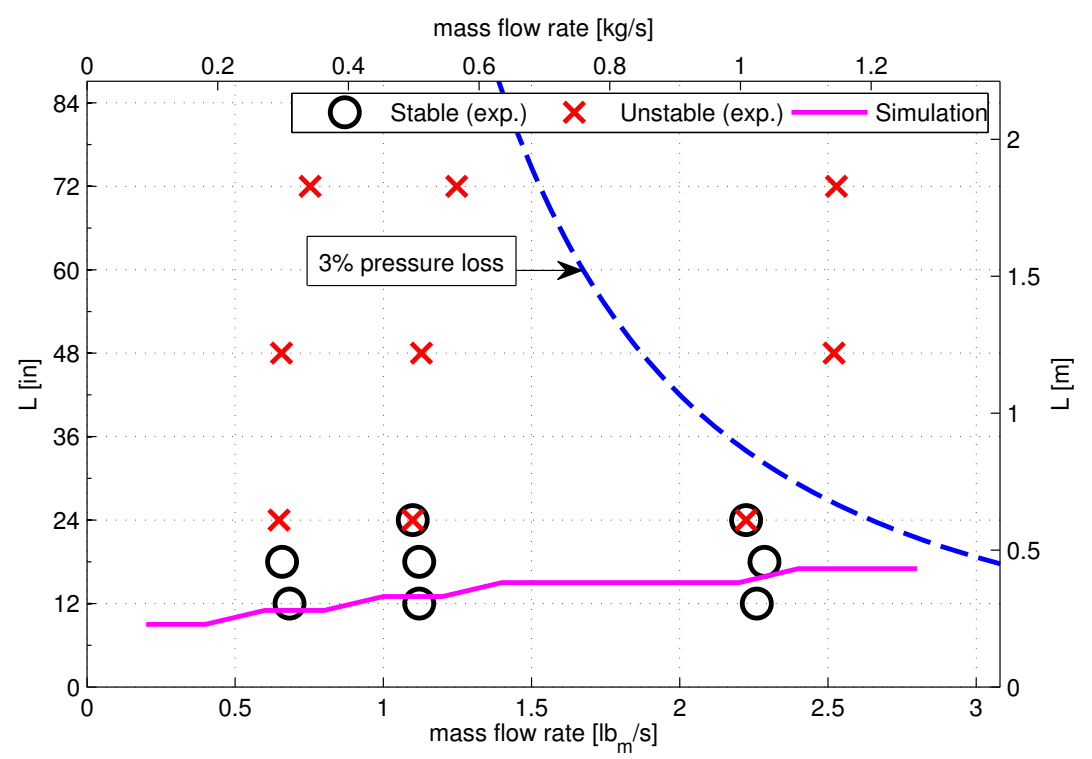

Figure 15: Stability map for valve 1E2, see text for details.

On the same diagram we have plot the results of each of the experimental test runs, categorizing each run as either stable (marked with a red circle) or unstable (a red cross). In cases where different stability characteristics were found upon valve opening and on closing, this is marked by both a circle and a cross.

Note the broad agreement between the location and the trend of the instability curve between experiments and simulations. We should stress here that there has been no parameter fitting whatsoever to achieve this result.

Superimposed on each stability chart is the $3 \%$ inlet pressure loss criterion derived from (16). Note how this curve has very little correlation with the flutter instability boundary we have computed. This is hardly surprising, since this curve is supposed to represent a completely different effect, namely a threshold beyond which a valve may be susceptible to low frequency cycling, rather than the onset of a self-excited high-frequency Hopf bifurcation associated with the quarter-wave mode in the pipe. 


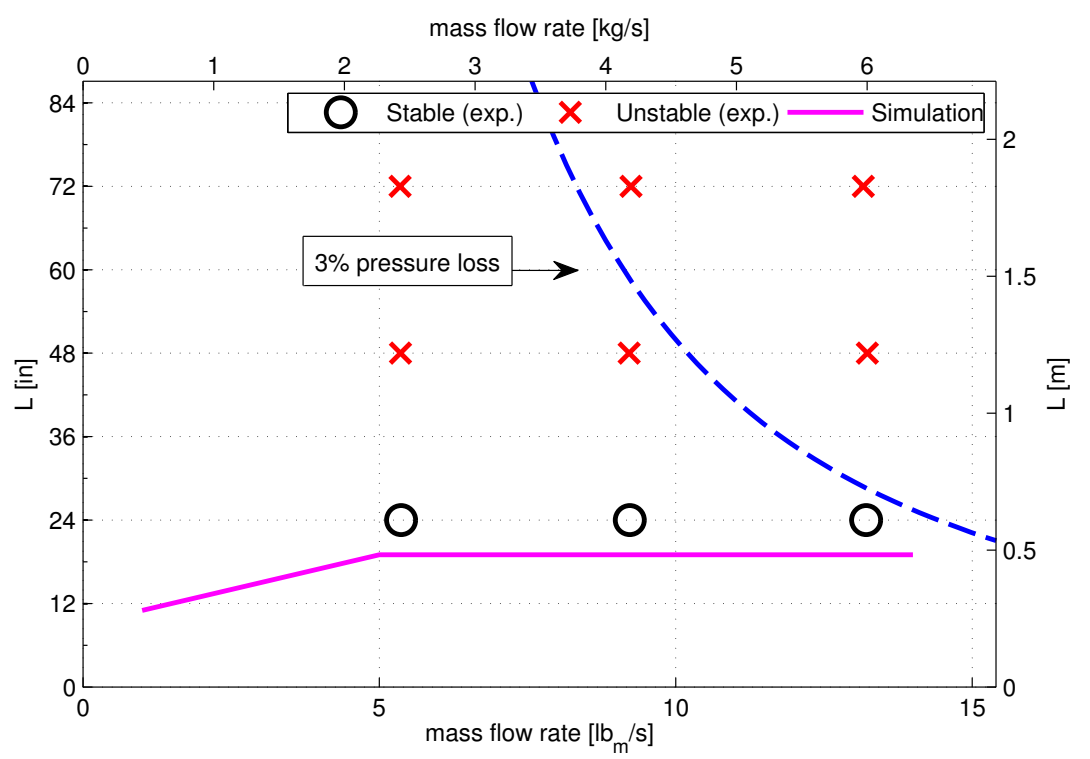

Figure 16: Similar to Fig. 15 but for valve 2J3.

\section{Summary and outlook}

In summary, we have produced a detailed, validated, fully parametrized mathematical model of a direct spring operated pressure relief valve connected by a pipe to a tank. There is a good match between simulation outputs from this model and detailed experimental measurements on three different commercially available valves.

Within this model, we have found that the effects of line pressure loss are not important, although running the valve at low flow rates can result in cycling in which the valve is prone to low-frequency oscillation with the valve closed for a significant proportion of each period. Such instabilities are not likely to be damage-inducing provided the valve opening itself is stable.

We have also found that the design of the valve can itself cause a form of static instability, in which the valves position jumps. These jumps can be predicted by analyzing the new concept we have introduced here of the effective-area versus lift curve. A simple criterion that compares the slope of this curve to the valve stiffness can be used to predict these jump points. However, such instabilities do not lead to flutter or chatter behaviour, but can contribute to cycling, or to rapid transitions between stable and unstable 


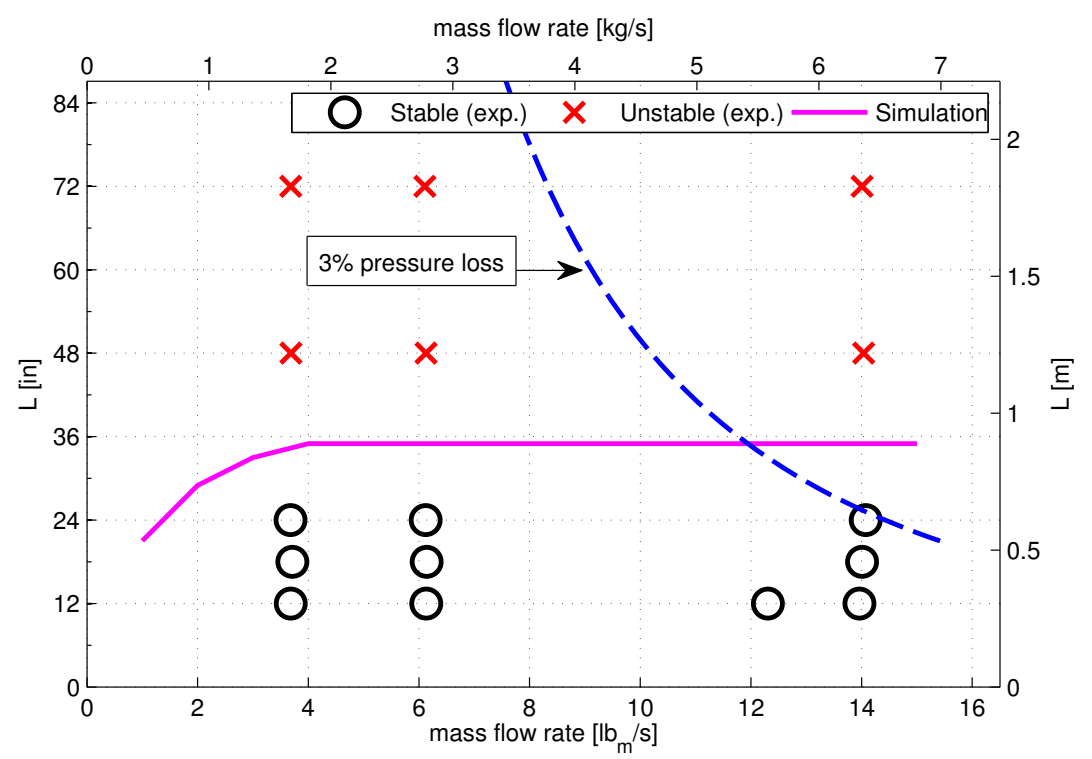

Figure 17: Similar to Fig. 15 but for valve 3L4.

operation.

Much more serious is a self-excited instability (a Hopf bifurcation) due to a coupling between the valve and a pressure wave in the inlet pipe. We should stress that the observed instability is not in any sense due to resonant coupling between the valve and the pipe's natural frequency. Rather it is a fully coupled mode of instability in which the valve in effect provides negative damping to the lowest-frequency wave within the pipe, namely the quarter wave.

Moreover, we have been able to trace, both theoretically and in experiments, a critical curve in pipe-length vs. mass flow rate, beyond which the instability occurs. This we can think of as a neutral stability curve. Beneath this curve, the valve effectively provides damping to the acoustic pipe mode; whereas above it, we have the negative damping. Note the shape of the neutral stability curve is that for a pipe of a given length, the instability is triggered upon reducing the mass flow rate. For longer pipes (equivalently, lower mass flow-rates) the amplitude of the valve oscillations can become so-extreme that the valve first grazes with its seat. This leads to impacting motion, or chatter. As first identified in Licsko et al. (2009), in terms of 
dynamical systems theory, the transition to impacting motion is an example of a grazing bifurcation.

A much more delicate question is how the instability may be prevented in practice. Any in-service pressure-relief event is in truth a transient operation, and there may be insufficient time at a given mass-flow rate in order to trigger an instability. In particular, the aim of any operation would be to be at a flow rate beyond the critical mass flow-rate for stability for the given inlet pipe length. This suggests that rapid opening of the valve to a sufficiently high flow rate, or rapid closing from a high flow rate to a low one may be one way of avoiding instability in practice. Indeed, this was effectively observed in our experimental data, in which we found different thresholds for instabilities upon opening the valve than upon closing.

One thing to note from the study by Izuchi (2010) is that friction effects for very long pipes can have a stabilizing influence. We have found no direct evidence for this effect in neither our test data nor simulations. However, it may be that we need to investigate much longer inlet pipes to observe this effect. However, in longer pipes, other pipe modes may additionally become relevant, as shown in Hayashi (1995) and Botros et al. (1997), who predict further instabilities for longer pipes. This issue is worthy of further investigation.

We should stress that our conclusions on the mechanisms of instability are strictly speaking only valid for the particular valves tested in the particular parameter region studied. Nevertheless, the mechanisms of instability we describe appear quite general, as evidenced by their occurrence for three separate valves we have tested, and preliminary modeling studies that suggest that they occur for a wide range of different parameter values. Moreover, our mathematical model approach is in principle easily extensible to capture for example effects of back pressure, pilot-operated valves, and relief valves in liquid. Each of these will be addressed in future work. Indeed, preliminary analysis relevant to liquids (based on the study in Hös and Champneys (2012)) has revealed another mode of instability that involves the valve's resonant frequency, without exciting quarter waves within the pipe.

\section{Acknowledgements}

This project was financially supported by Csaba Hős's Bolyai fellowship of the Hungarian Academy of Sciences. 


\section{References}

Askari, E., Jeong, K.H., Amabili, M., 2013. Hydroelastic vibration of circular plates immersed in a liquid-filled container with free surface. Journal of Sound and Vibration .

Bazsó, C., Champneys, A., Hős, C., 2013a. Bifurcation analysis of a simplified model or a pressure relief valve attached to a pipe. Submitted to SIAM J. Applied Dynamical Systems.

Bazsó, C., Champneys, A., Hős, C., 2013b. Model reduction of a direct spring-loaded pressure relief valve with upstream pipe. Submitted to IMA J. Applied Math.

Bazsó, C., Hős, C., 2013a. An experimental study on the stability of a direct spring loaded poppet relief valve. Preprint.

Bazsó, C., Hős, C., 2013b. On the static instability of liquid poppet valves. Submitted to Periodica Polytechnica Mechanical Engineering.

Bean, H., 1971. Report of ASME Research Committee on Fluids meters. Technical Report. ASME.

Beune, A., 2009. Analysis of high-pressure safety valves. Ph.D. thesis. PhD thesis. Eindhoven, The Netherlands: Eindhoven University of Technology.

Botros, K., Dunn, G., Hrycyk, J., 1997. Riser-relief valve dynamic interactions. Journal of Fluids Engineering 119, 671-679.

Cebeci, T., Shao, J.P., Kafyeke, F., Laurendeau, E., 2005. Computational fluid dynamics for engineers: from panel to Navier-Stokes methods with computer programs. Springer.

Chabane, S., Plumejault, S., Pierrat, D., Couzinet, A., Bayart, M., 2009. Vibration and chattering of conventional safety relief valve under built up back pressure, in: Proceedings of the 3rd IAHR International Meeting of the WorkGroup on Cavitation and Dynamic Problems in Hydraulic Machinery and Systems, pp. 281-294.

D’Netto, W., Weaver, D., 1987. Divergence and limit cycle oscillations in valves operating at small openings. Journal of Fluids and Structures 1, $3-18$. 
Francis, J., Betts, P., 1998. Backpressure in a high-lift compensated pressure relief valve subject to single phase compressible flow. Journal of Loss Prevention in the Process Industries 11, 55 - 66. doi:10.1016/S09504230(98)00003-5.

Funk, J., 1964. Poppet valve stability. Journal of Basic Engineering 86, 207.

Green, W., Woods, G., 1973. Some causes of chatter in direct acting spring loaded poppet valve, in: The 3rd International Fliud Power Symposium, Turin.

Habing, R., Peters, M., 2006. An experimental method for validating compressor valve vibration theory. Journal of Fluids and Structures 22, 683697.

Hayashi, S., 1995. Instability of poppet valve circuit. JSME International Journal. Ser. C, Dynamics, Control, Robotics, Design and Manufacturing $38,357-366$.

Hayashi, S., Hayase, T., Kurahashi, T., 1997. Chaos in a hydraulic control valve. Journal of Fluids and Structures 11, $693-716$.

Hös, C., Champneys, A., 2012. Grazing bifurcations and chatter in a pressure relief valve model. Physica D: Nonlinear Phenomena 241, 2068-2076. doi:10.1016/j.physd.2011.05.013.

Ih, J.G., 1993. On the inertial end correction of resonantors. Acustica 78, $1-15$.

Izuchi, H., 2010. Stability analysis of safety valve. American Institute of Chemical Engineers, 10th Topical Conference on Natural Gas Uitilization ISBN: 9781617384417.

Kasai, K., 1968. On the stability of a poppet valve with an elastic support : 1st report, considering the effect of the inlet piping system. Bulletin of JSME 11, 1068-1083.

Khalak, A., Williamson, C., 1997. Fluid forces and dynamics of a hydroelastic structure with very low mass and damping. Journal of Fluids and Structures 11, 973-982. 
Kuznetsov, Y., 2004. Elements of applied bifurcation theory. Springer-Verlag.

Licsko, G., Champneys, A., Hős, C., 2009. Nonlinear analysis of a single stage pressure relief valve. Int. J. Appl. Math 39, 12-26.

MacLeod, G., 1985. Safety valve dynamic Instability:An analysis of chatter. Journal of Pressure Vessel Technology 107, 172-177.

Madea, T., 1970a. Studies on the dynamic characteristic of a poppet valve: 1st report, theoretical analysis. Bulletin of JSME 13, 281-289.

Madea, T., 1970b. Studies on the dynamic characteristics of a poppet valve: 2nd report, experimental analysis. Bulletin of JSME 13, 290-297.

McCloy, D., McGuigan, R., 1964. Some static and dynamic characteristics of poppet valves, in: Proceedings of the Institution of Mechanical Engineers, Prof Eng Publishing. pp. 199-213.

Misra, A., Behdinan, K., Cleghorn, W., 2002. Self-excited vibration of a control valve due to fluid-structure interaction. Journal of Fluids and Structures 16, $649-665$.

Moussou, P., Gibert, R., Brasseur, G., Teygeman, C., Ferrari, J., Rit, J., 2010. Instability of pressure relief valves in water pipes. Journal of Pressure Vessel Technology 132.

Nayfeh, A., Bouguerra, H., 1990. Non-linear response of a fluid valve. International Journal of Non-Linear Mechanics 25, 433-449.

Song, X.G., Park, Y.C., Park, J.H., 2011. Blowdown prediction of a conventional pressure relief valve with a simplified dynamic model. Mathematical and Computer Modelling 57, 279-288.

Thomann, H., 1976. Oscillations of a simple valve connected to a pipe. Zeitschrift f ur Angewandte Mathematik und Physik (ZAMP) 27, 23-40.

Vaughan, N., Johnston, D., Edge, K., 1992. Numerical simulation of fluid flow in poppet valves. Proceedings of the Institution of Mechanical Engineers 206, 119-127. 
Warren, M., 1983. Appropriate boundary conditions for the solution of the equations of unsteady one-dimensional gas flow by the Lax-Wendroff method. International Journal of Heat and Fluid Flow 4, 53-59.

Ye, Q., Chen, J., 2009. Dynamic analysis of a pilot-operated two-stage solenoid valve used in pneumatic system. Simulation Modelling Practice and Theory 17, 794-816.

Zucker, D., Biblarz, O., 2002. Fundamentals of Gas Dynamics. New York: John Wiley and Sons.

Zucrow, M.J., Hoffman, J.D., 1976. Gas dynamics. volume 1. New York: John Wiley and Sons, 1976.

Zung, P., Perng, M., 2002. Nonlinear dynamic model of a two-stage pressure relief valve for designers. Journal of dynamic systems, measurement, and control 124, 62-66. 